\title{
First case of non-exposed endoscopic wall-inversion surgery with sentinel node basin dissection for early gastric cancer
}

\author{
Osamu Goto $\cdot$ Hiroya Takeuchi $\cdot$ Hirofumi Kawakubo $\cdot$ Motoki Sasaki $\cdot$ \\ Tatsuo Matsuda $\cdot$ Satoru Matsuda $\cdot$ Yu Kigasawa $\cdot$ Yoshie Kadota $\cdot$ \\ Ai Fujimoto $\cdot$ Yasutoshi Ochiai $\cdot$ Joichiro Horii $\cdot$ Toshio Uraoka $\cdot$ \\ Yuko Kitagawa $\cdot$ Naohisa Yahagi
}

Received: 26 March 2014/ Accepted: 14 July 2014/Published online: 3 August 2014

(C) The International Gastric Cancer Association and The Japanese Gastric Cancer Association 2014

\begin{abstract}
Introduction Non-exposed endoscopic wall-inversion surgery (NEWS) is a novel technique of endoscopic fullthickness resection without transluminal access mainly designed to treat gastric cancer. Here, we report a successful case of NEWS with sentinel node basin dissection (SNBD) for early gastric cancer (EGC) with the risk of lymph node metastasis.

Patient and methods A 55-year-old female patient with a 2-cm, diffuse-type intramucosal EGC with ulceration was referred to our hospital for a less invasive gastrectomy based on sentinel node navigation surgery. After obtaining informed consent, NEWS with SNBD was applied. After placing mucosal markings, indocyanine green solution was injected endoscopically into the submucosa around the lesion to examine sentinel nodes (SNs). The SN basin (the area of the left gastric artery), including three stained $\mathrm{SNs}(\# 3)$, was dissected, and an intraoperative pathological diagnosis confirmed that no metastasis had occurred. Subsequently, NEWS was performed for the primary lesion. Serosal markings were placed laparoscopically, submucosal injection was added endoscopically, and circumferential sero-muscular incision and suturing were performed laparoscopically, with the lesion inverted
\end{abstract}

O. Goto · M. Sasaki · A. Fujimoto · Y. Ochiai - J. Horii ·

T. Uraoka $\cdot$ N. Yahagi

Division of Research and Development for Minimally Invasive

Treatment, Cancer Center, Keio University, School of Medicine,

Tokyo, Japan

H. Takeuchi $(\bowtie) \cdot$ H. Kawakubo · T. Matsuda - S. Matsuda .

Y. Kigasawa · Y. Kadota · Y. Kitagawa

Department of Surgery, Keio University, School of Medicine, 35

Shinanomachi, Shinjuku-ku, Tokyo 160-8582, Japan

e-mail: htakeuchi@a6.keio.jp toward the inside of the stomach. Finally, the circumferential mucosal incision was performed, and the lesion was retrieved perorally.

Results The operation was finished in 270 min without complications. The patient was uneventfully discharged 10 days after the procedure. The final pathological diagnosis was coincident with the pre- and intraoperative assessment.

Conclusions We demonstrated the feasibility and safety of NEWS with SNBD with a favorable result. This surgical concept is expected to become a promising, minimally invasive, function-preserving surgery to cure cases of EGC that are possibly node-positive.

Keywords Minimally invasive surgery $\cdot$ Local resection . Sentinel node navigation surgery $\cdot$ Early gastric cancer

\section{Introduction}

Endoscopic full-thickness resection (EFTR) with or without laparoscopic assistance is a promising method to minimize a resection area according to a lesion size [1-4]. Because a demarcation line of the lesion can be clearly visualized from the inside of the lumen by using endoscopy, the resection area can be optimally determined via this technique. However, intentional perforation is necessary during the procedure, which might cause peritonitis due to leakage of intraluminal fluid. Furthermore, because of the risk of tumor seeding especially in the case of cancers, the indication of EFTR is limited to submucosal tumors only.

Non-exposed endoscopic wall-inversion surgery (NEWS) was developed as a new full-thickness resection technique without intentional perforation mainly aimed at 

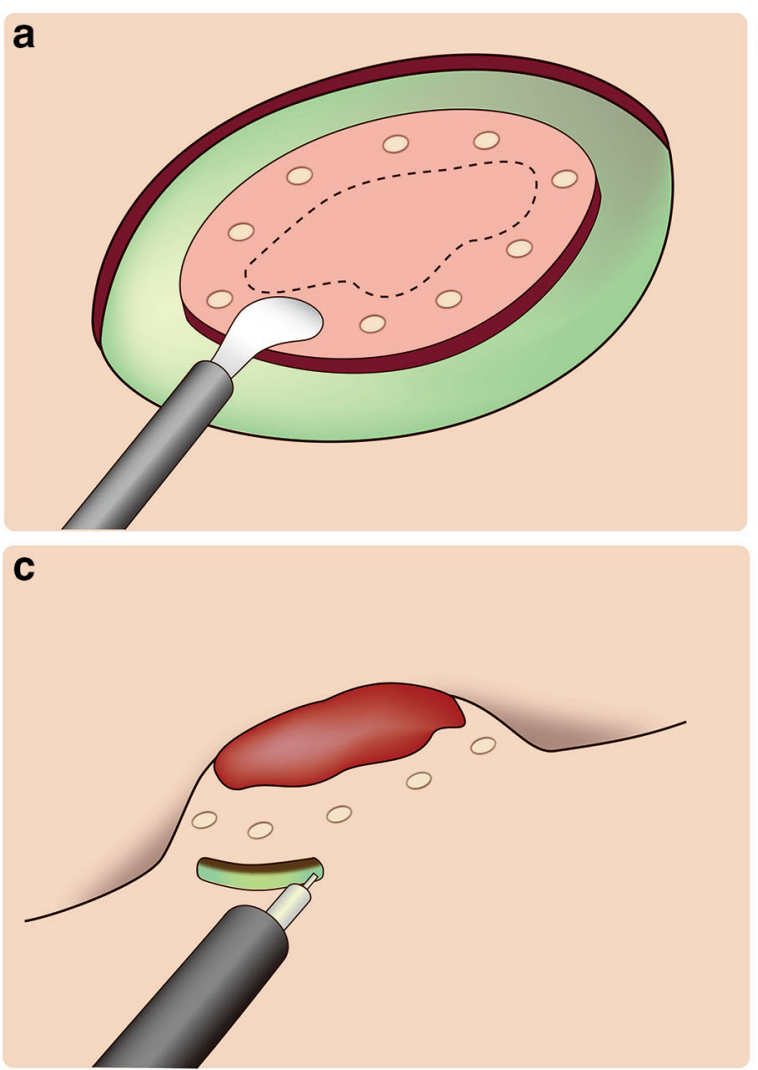

Fig. 1 Illustration of the non-exposed endoscopic wall-inversion surgery (NEWS): a Laparoscopic circumferential sero-muscular incision after endoscopic submucosal injection; b laparoscopic seromuscular suturing and inversion of the lesion. During the suturing, a

being a minimally-invasive surgery for gastric cancer (Fig. 1) [5-7]. The feasibility of NEWS combined with the concept of sentinel node navigation surgery (SNNS) [8,9], which can minimize the area of lymphadenectomy, has been already demonstrated in a porcine survival study as an ideal method of function-preserving surgery for early gastric cancer (EGC) [10]. We herein report a first clinical case of NEWS with sentinel node basin dissection (SNBD) for possibly node-positive EGC.

\section{Patient and methods}

A 55-year-old female underwent an upper gastrointestinal barium X-ray during a health check that showed a regional irregular surface on the lesser curvature of the lower gastric body. Esophagogastroduodenoscopy revealed a $2-\mathrm{cm}$ depressed-type EGC, and the pathological findings of the biopsy specimens indicated diffuse-type adenocarcinoma (Fig. 2a). Although intramucosal cancer was suspected, endoscopic resection was not recommended because of the existence of ulcerative findings, which implied possible lymph node metastasis [11]. A computed tomography (CT)

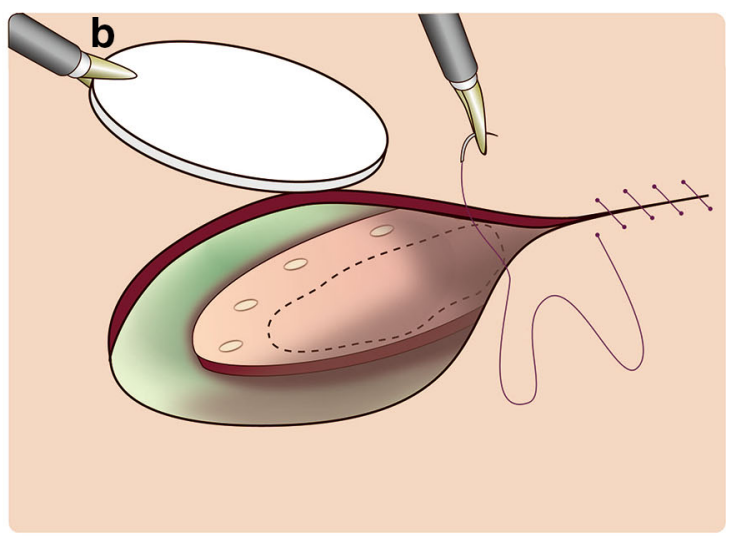

d

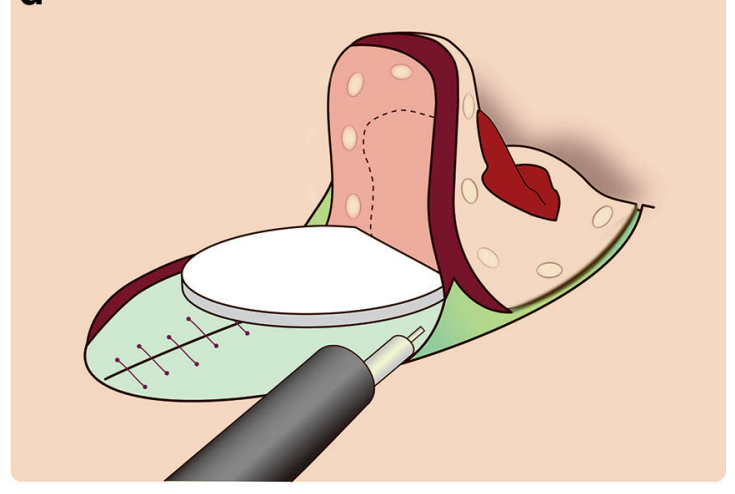

surgical sponge is inserted as a spacer. c Endoscopic circumferential mucosal incision; d Endoscopic incision of a remnant submucosal tissue

scan showed no evidence of metastasis to the lymph nodes or other organs. The patient was referred to a surgeon at another hospital and was informed about several options of surgical methods, e.g., distal gastrectomy, laparoscopyassisted distal gastrectomy, and laparoscopic-assisted pylorus-preserving gastrectomy. However, she desired a less invasive surgery than all of the above options, and was referred to our hospital for more minimally invasive surgery by SNNS. She was thoroughly informed of the NEWS procedure with SNBD as a clinical trial, as well as the other options mentioned above, and agreed to this new method.

Under general anesthesia, the lesion was clearly observed by conventional endoscopy, narrow band imaging with magnifying endoscopy, and chromoendoscopy to demarcate the tumor margin and to decide the resection area, and several mucosal markings were placed approximately $5-10 \mathrm{~mm}$ outside the lesion using the tip of a 2.0mm Dual knife (KD-650L; Olympus Medical Systems, Co., Ltd., Tokyo, Japan) (Fig 2b). Subsequently, one camera port at the umbilicus and four trocars were inserted at four quadrants of the upper left, upper right, lower left, and lower right, respectively. At each of the four quadrants around the lesion, $0.5 \mathrm{ml}$ of indocyanine green (ICG) 

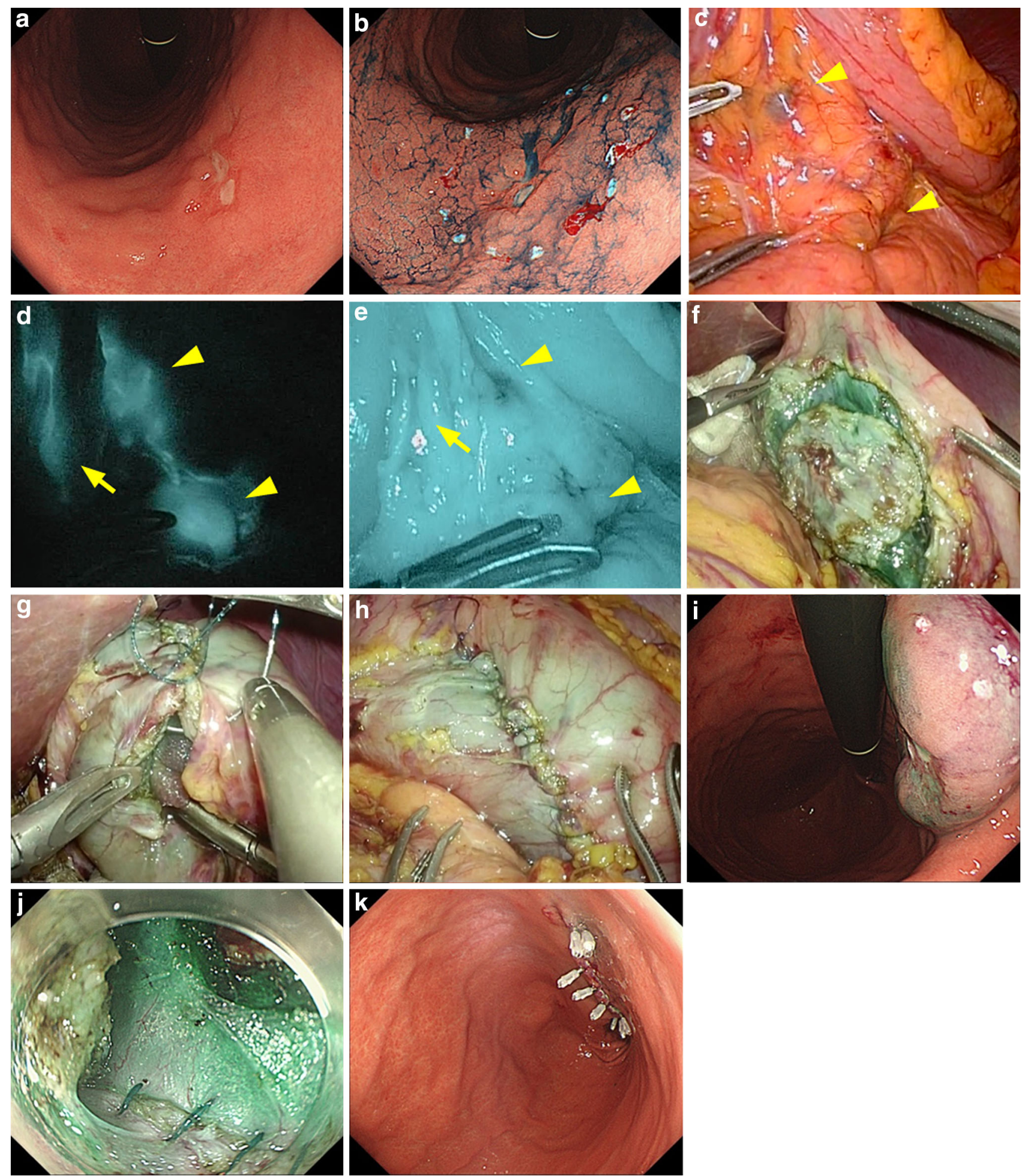

Fig. 2 Procedure of non-exposed endoscopic wall-inversion surgery (NEWS) with sentinel node basin dissection (SNBD). a Diffuse-type early gastric cancer $(0-$ IIc + III) is located at the lesser curvature of the lower gastric body. b Mucosal markings were placed after precise observation of a demarcation line. c A sentinel node (\#3a) stained by indocyanine green is identified (arrowheads). These nodes and the primary lesion (arrow) are visualized more clearly by $\mathbf{d}$ a near-

infrared ray image and $\mathbf{e}$ an indocyanine green fluorescence image. f Circumferential sero-muscular incision and slight submucosal dissection. g The lesion was inverted toward the inside with a surgical spacer. h Sero-muscular linear suturing is finished. i Protruded lesion. j A suturing line is seen during endoscopic mucosal incision. $\mathbf{k}$ Mucosal clipping after the resection 

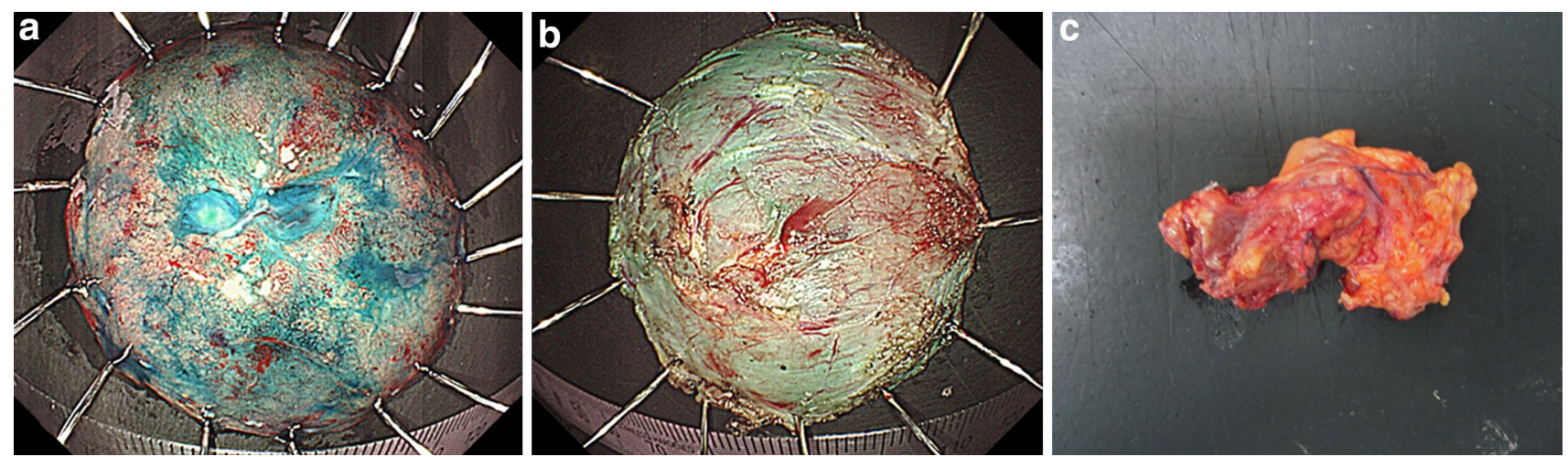

Fig. 3 Resected specimens: a mucosal side and $\mathbf{b}$ serosal side of the primary lesion. Full layers can be resected without discordance between the two sides. $\mathbf{c}$ Resected sentinel node basin (the area of left gastric artery) including sentinel nodes (\#3a)

solution $(5 \mathrm{mg} / \mathrm{ml})$ was injected into the submucosal layer endoscopically to detect sentinel nodes. After $10 \mathrm{~min}$ of injection, two stained lymph nodes (\#3a) were identified as SNs laparoscopically (Fig. 2c). These lymph nodes were confirmed by ICG infrared/fluorescence imaging using the Near-Infrared Fluorescence Laparoscopic System (Olympus) (Fig. 2d, e). Subsequently, an SN basin (\#1, \#3a, and \#7; the area of the left gastric artery) including these SNs was dissected. After confirming no metastasis at the SNs by intraoperative pathological diagnosis using a frozen section, NEWS was performed for the primary lesion. In the placing of serosal markings using a spatula-type electrode (A6284; Olympus), mucosal markings were protruded toward the outside using the Dual Knife for the laparoscopist to recognize the location of these markings. A transparently-visible shadow of the device from the opposite side was also used to make the serosal markings. Sodium hyaluronate solution (MucoUp ${ }^{\circledR}$; Seikagaku Corporation, Tokyo, Japan) with a small amount of ICG was additionally injected endoscopically into the submucosa around the circumference of the lesion, followed by a laparoscopic circumferential sero-muscular incision $5 \mathrm{~mm}$ outside the serosal markings (Fig. 1a, Fig.2f). In order to avoid perforating the mucosa from the outside, the seromuscular layer was cut carefully up to the level of the submucosa stained with green. After deeper cutting of the submucosa toward the outside to create a flap, the seromuscular layers were linearly sutured, with the lesion inverted toward the inside of the stomach. On the way to the suturing, a laparoscopic surgical spacer $\left(\right.$ Securea ${ }^{\circledR}$; Hogy Medical Co., Ltd., Tokyo, Japan) that was cut elliptically according to the size of the lesion was inserted between the serosal layer of the inverted lesion and the suture layer, in order to provide a counter-traction to the mucosa and to prevent cutting the suture during the subsequent endoscopic procedure (Fig. 1b, Fig.2g, h). Finally, the circumferential mucosal incision and subsequent incision of the remnant submucosal tissue was made $5 \mathrm{~mm}$ outside of the mucosal markings around the inverted lesion using the Dual knife, while the inserted spacer was dug out (Fig. 1c, d, Fig.2i, j). The detached lesion and the spacer were retrieved perorally, and the mucosal edges were closed with several endoscopic clips (HX-610-90L; Olympus) (Fig. 2k). After confirming no air leakage by pooling with normal saline on the serosal side of the suture, and then collecting $10 \mathrm{ml}$ of peritoneal lavage fluid for cytology, endoscopy and laparoscopic devices were withdrawn and scars were sutured.

\section{Results}

The operation was finished in 270 min without complications, and estimated blood loss was less than $10 \mathrm{ml}$. The postoperative course was also quite favorable without adverse events, and the patient was discharged on postoperative day (POD) 10. The maximum body temperature, white blood cell count, and C-reactive protein count were $37.5^{\circ}$ Celsius on POD, $0.4600 / \mathrm{mm}^{3}$ on POD 1 , and $0.97 \mathrm{mg} / \mathrm{dl}$ on POD 2, respectively. The final diagnosis was poorly differentiated adenocarcinoma (por $>$ sig), $20 \times 18 \mathrm{~mm}$ in tumor size $(38 \times 32 \mathrm{~mm}$ in specimen size), Type 0-IIc + III, pT1a (M), and no angiolymphatic invasion (Fig. 3a, b). The surgical margin was tumor negative and no metastasis of carcinoma was found in the dissected lymph nodes (\#3a [0/7], \#7 [0/4]) (Fig. 3c). No tumor cells were found in the collected peritoneal lavage fluid. In the first outpatient follow-up appointment one month after the operation, the patient reported no apparent discomfort about food intake.

\section{Discussion}

We successfully demonstrated that NEWS with SNBD was feasible and could be safely introduced for humans. To the 
best of our knowledge, this concept would be the most minimized gastrectomy and lymphadenectomy for possibly node-positive EGC.

In the current situation, EGC involving the possibility of lymph node metastasis necessitates wide resection of regional lymph nodes because we cannot know where cancer cells metastasize in a lymphatic network around the stomach. However, the rate of lymph node metastasis is only 10-20\% [12]. Accordingly, in 80-90\% of EGCs suspected of lymph node metastasis, unnecessary lymphadenectomy has to be performed, which has to be accompanied with unnecessarily wide gastrectomy to avoid necrosis of the gastric wall. The concept of SNNS enables us to minimize the area of lymphadenectomy by detecting lymphatic flows, which can also allow the area of gastrectomy to be minimized. We have already verified the feasibility of SNNS with a large amount of clinical cases [8].

A dual-tracer method, which was recommended for secure identification of SNs, was not applied in this case. Instead of adopting a radio-guided method with a radioactive tracer, we increased the diagnostic accuracy in detection of SNs by using ICG infrared/fluorescence imaging. Both imaging modalities facilitate the detection of SNs by enhancing the visualization of ICG-stained SNs as well as lymphatic vessels [13, 14].

In current function-preserving surgeries such as laparoscopic local resection or segmental gastrectomy, the approach of gastrectomy is only from the outside of the stomach, in which the demarcation line of the tumor cannot be visualized at the phase of resection. Therefore, the operator cannot avoid a wider resection of the stomach than is desired to prevent a positive surgical margin. The method of full-layer resection for gastric cancer with nonexposure known as CLEAN-NET is another fascinating technique of non-exposed local resection [15], but there is still room for minimizing the resection area because the lesion is finally resected laparoscopically. Furthermore, unlike other EFTR techniques irrespective of laparoscopic assistance, NEWS does not need intentional perforation, which enables us to apply this technique to cancers without a risk of iatrogenic dissemination. Therefore, NEWS combined with SNBD would be the best resection technique to minimize the resection area for $\mathrm{EGC}$ with negative SN metastasis. By introducing this technique, unnecessarily wide resection can be avoided and the stomach would be saved as much as possible in 80-90 \% of the EGC cases mentioned above.

Nunobe and colleagues introduced a modified method of laparoscopic and endoscopic cooperative surgery to avoid dissemination [16]. In this technique, a surrounding gastric wall of the lesion is hooked up with several strings through the abdominal wall in order to prevent a spill of gastric fluid into the abdominal space. However, the strings used for hooking go through the gastric wall, which might cause tumor implantation to the gastric or abdominal wall. Furthermore, laparoscopic devices can touch the exposed cancer cells during the procedure, which might cause dissemination or port-site metastasis. On the other hand, a gastric hole penetrating the full layer is not created during the NEWS procedure even though serosal incision is necessary, and hence, cancer cells, which are exposed to the mucosal surface, would be neither touched nor disseminated. Although these potential risks might become negligible statistically, NEWS is thought to be superior to this technique, at least theoretically.

The clinical benefit of this technique should be advocated for after accumulating additional patients. Estimation of long-term outcomes is also required to demonstrate its efficacy and safety, especially in terms of patient quality of life and the risk of recurrence. Further studies are needed to establish NEWS with SNBD as a standard procedure.

In conclusion, we demonstrated that the short-term outcomes of NEWS with SNBD were quite favorable in a single case of EGC. This method is expected to be recognized an ideal surgery for function-preserving gastrectomy for EGC with possible lymph node metastasis.

Acknowledgments O.G. was funded by a Grant-in-Aid for Young Scientists from the Ministry of Education, Culture, Sports, Science and Technology in Japan in 2013-2014 (Grant No. 25860558). We thank Isamu Shinohara and Miyuki Okabe (thanks to Corporation, Saitama, Japan) for creating the illustration of the NEWS procedure.

Conflict of interest No conflict of interest exists.

\section{References}

1. Hiki N, Yamamoto Y, Fukunaga T, Yamaguchi T, Nunobe S, Tokunaga $\mathrm{M}$, et al. Laparoscopic and endoscopic cooperative surgery for gastrointestinal stromal tumor dissection. Surg Endosc. 2008;22:1729-35.

2. Abe N, Takeuchi H, Yanagida O, Masaki T, Mori T, Sugiyama $\mathrm{M}$, et al. Endoscopic full-thickness resection with laparoscopic assistance as hybrid NOTES for gastric submucosal tumor. Surg Endosc. 2009;23:1908-13.

3. Schlag C, Wilhelm D, von Delius S, Feussner H, Meining A. EndoResect study: endoscopic full-thickness resection of gastric subepithelial tumors. Endoscopy. 2013;45:4-11.

4. Chiu PW, Phee SJ, Wang Z, Sun Z, Poon CC, Yamamoto T, et al. Feasibility of full-thickness gastric resection using master and slave transluminal endoscopic robot and closure by overstitch: a preclinical study. Surg Endosc. 2014;28:319-24.

5. Goto O, Mitsui T, Fujishiro M, Wada I, Shimizu N, Seto Y, et al. New method of endoscopic full-thickness resection: a pilot study of non-exposed endoscopic wall-inversion surgery in an ex vivo porcine model. Gastric Cancer. 2011;14:183-7.

6. Mitsui T, Goto O, Shimizu N, Hatao F, Wada I, Niimi K, et al. Novel technique for full-thickness resection of gastric malignancy: feasibility of nonexposed endoscopic wall-inversion surgery (NEWS) in porcine models. Surg Laparosc Endosc Percutan Tech. 2013;23:e217-21. 
7. Mitsui T, Niimi K, Yamashita H, Goto O, Aikou S, Hatao F, et al. Non-exposed endoscopic wall-inversion surgery as a novel partial gastrectomy technique. Gastric Cancer. 2013 [Epub ahead of print].

8. Kitagawa Y, Takeuchi H, Takagi Y, Natsugoe S, Terashima M, Murakami N, et al. Sentinel node mapping for gastric cancer: a prospective multicenter trial in Japan. J Clin Oncol. 2013;31: 3704-10.

9. Takeuchi H, Kitagawa Y. New sentinel node mapping technologies for early gastric cancer. Ann Surg Oncol. 2013;20:522-32.

10. Goto O, Takeuchi H, Kawakubo H, Matsuda S, Kato F, Sasaki M, et al. Feasibility of non-exposed endoscopic wall-inversion surgery with sentinel node basin dissection as a new surgical method for early gastric cancer: a porcine survival study. Gastric Cancer. 2014 [in press].

11. Association Japanese Gastric Cancer. Japanese gastric cancer treatment guidelines 2010 (ver. 3). Gastric Cancer. 2011;14: 113-23.

12. Gotoda T, Yanagisawa A, Sasako M, Ono H, Nakanishi Y, Shimoda $\mathrm{T}$, et al. Incidence of lymph node metastasis from early gastric cancer: estimation with a large number of cases at two large centers. Gastric Cancer. 2000;3:219-25.

13. Nimura H, Narimiya N, Mitsumori N, Yamazaki Y, Yanaga K, Urashima M. Infrared ray electronic endoscopy combined with indocyanine green injection for detection of sentinel nodes of patients with gastric cancer. Br J Surg. 2004;91:575-9.

14. Miyashiro I, Miyoshi N, Hiratsuka M, Kishi K, Yamada T, Ohue $\mathrm{M}$, et al. Detection of sentinel node in gastric cancer surgery by indocyanine green fluorescence imaging: comparison with infrared imaging. Ann Surg Oncol. 2008;15:1640-3.

15. Inoue H, Ikeda H, Hosoya T, Yoshida A, Onimaru M, Suzuki M, et al. Endoscopic mucosal resection, endoscopic submucosal dissection, and beyond: full-layer resection for gastric cancer with nonexposure technique (CLEAN-NET). Surg Oncol Clin N Am. 2012;21:129-40.

16. Nunobe $S$, Hiki N, Gotoda T, Murao T, Haruma K, Matsumoto H, et al. Successful application of laparoscopic and endoscopic cooperative surgery (LECS) for a lateral-spreading mucosal gastric cancer. Gastric Cancer. 2012;15:338-42. 